\title{
Reviewing the prognostic factors in myelomeningocele
}

\author{
Tatiana Protzenko, MD, MSc, ${ }^{1}$ Antônio Bellas, MD, MSc, ${ }^{1}$ Marcelo Sampaio Pousa, MD, ${ }^{2}$ \\ Michelle Protzenko, MD, ${ }^{2}$ Juliana Marin Fontes, MD, ${ }^{3}$ Anne Margareth de Lima Silveira, MD, ${ }^{1}$ \\ Cynthia Amaral Sá, MD, ${ }^{4}$ José Paulo Pereira Jr., MD, PhD, ${ }^{4}$ Renato Manganelli Salomão, MD, ${ }^{5}$ \\ José Francisco M. Salomão, MD, PhD, ${ }^{1}$ and Saint Clair dos Santos Gomes Jr., PhD ${ }^{6}$
}

\begin{abstract}
${ }^{1}$ Department of Pediatric Surgery, Division of Pediatric Neurosurgery; ${ }^{2}$ Clinical Research Unit; ${ }^{3}$ Department of Pediatric Urodynamics and Dysfunctional Voiding; ${ }^{4}$ Department of Fetal Medicine and Neonatology; ${ }^{5}$ Federal University of Rio de Janeiro State, Rio de Janeiro; and ${ }^{6} \mathrm{Clinical}$ Research Unit, Fernandes Figueira National Institutes of Health for Women, Children, and Adolescents, Oswaldo Cruz Foundation (IFF-Fiocruz), Rio de Janeiro, Brazil
\end{abstract}

\begin{abstract}
OBJECTIVE The goal of this study was to analyze the factors that have an impact on morbidity and mortality in patients with myelomeningocele (MMC).

METHODS A retrospective cohort study was conducted to analyze factors associated with MMC that influence the morbidity and mortality of the disease. Data were collected from medical records of children who underwent the primary repair of MMC at the Fernandes Figueira Institute-Oswaldo Cruz Foundation (IFF-Fiocruz) between January 1995 and January 2015, with a minimum follow-up of 1 year. The following variables were analyzed: demographic characteristics (gestational age, sex, and birth weight); clinical features (head circumference at birth, anatomical and functional levels of MMC, hydrocephalus, symptomatic Chiari malformation type II, neurogenic bladder, and urinary tract infection [UTI]); and surgical details such as timing of repair of MMC, age at first shunt placement, shunt surgery modality (elective or emergency), concurrent surgery (correction of MMC and shunt insertion in the same surgical procedure), incidence and cause of shunt dysfunction, use of external ventricular drain, transfontanelle puncture, surgical wound complications prior to shunting, and endoscopic treatment of hydrocephalus.

RESULTS A total of 231 patients with MMC were included in the analysis. Patients were followed for periods ranging from 1 to 20 years, with a mean of 6.9 years. The frequency of shunt placement was observed mainly among patients with MMC at the highest spinal levels $(p<0.01)$. The main causes of morbidity and mortality in patients with MMC were shunt failures, diagnosed in 91 of 193 cases (47.2\%) of hydrocephalus, and repeated UTIs, in 129 of 231 cases (55.8\%) of MMC; these were the main causes of hospitalization and death. Head circumference $\geq 38 \mathrm{~cm}$ at birth was found to be a significant risk factor for shunt revision ( $<<0.001 ; 95 \% \mathrm{Cl} 1.092-1.354)$. Also, the lumbar functional level of MMC was associated with less revision than upper levels $(p<0.014 ; 95 \% \mathrm{Cl} 0.143-0.805)$. There was a significant association between recurrent UTI and thoracic functional level.
\end{abstract}

CONCLUSIONS Macrocephaly at birth and higher levels of the defect have an impact on worse outcome and, therefore, are a challenge to the daily practice of pediatric neurosurgery.

https://thejns.org/doi/abs/10.3171/2019.7.FOCUS19462

KEYWORDS myelomeningocele; Chiari malformation type Il; hydrocephalus; cerebrospinal fluid shunt; spinal dysraphism

$\mathrm{T}$ HE worldwide incidence of neural tube defects (NTDs) ranges from 0.17 to 6.39 in 1000 live births. ${ }^{3}$ Myelomeningocele (MMC) is the most common NTD, characterized by a dorsal midline lesion composed of a neural plaque (placode) attached to adjoining dysplastic epithelial tissue. ${ }^{1}$ More than a congenital defect, it could lead to serious conditions such as Chiari malforma- tion type II (CM-II), hydrocephalus, motor disorders, neurogenic bladder, and orthopedic deformities.,20,31 These conditions have already been related to the morbidity and mortality of patients with MMC. In 1994, Kaufman et al. ${ }^{16}$ associated the inadequate follow-up of these patients with high morbidity. In 2003, Oakeshott and Hunt reported the association of childhood neurological deficits with the

ABBREVIATIONS CM-II = Chiari malformation type II; ETV = endoscopic third ventriculostomy; IFF-Fiocruz = Fernandes Figueira Institute-Oswaldo Cruz Foundation; $\mathrm{MMC}=$ myelomeningocele; $\mathrm{NTD}=$ neural tube defect; $\mathrm{PFD}=$ posterior fossa decompression; UTI = urinary tract infection.

SUBMITTED June 1, 2019. ACCEPTED July 30, 2019.

INCLUDE WHEN CITING DOI: 10.3171/2019.7.FOCUS19462. 
degree of mobility, independence, and even the patient's blood pressure. ${ }^{24}$ Also, in this series, death was related to cardiorespiratory or renal failure. ${ }^{13}$

In the early 1950s, the survival rate of individuals with MMC did not exceed $10 \%{ }^{20}$ Recently, with the great advances in the treatment of complications, a large number of children survive and reach adulthood., ${ }^{3,4,25}$ Even so, despite the decline in incidence and mortality, MMC continues to be a major cause of chronic disability. ${ }^{4}$ Therefore, the optimal treatment of patients with MMC requires multidisciplinary care to prevent, monitor, and treat potential complications that may affect function, quality of life, and survival. In this context, knowledge of the prognostic factors becomes fundamental. The aim of our study was to analyze the factors that impact morbidity and mortality in patients with MMC.

\section{Methods}

We conducted a retrospective study of all 384 patients who underwent the primary repair of MMC between January 1995 and January 2015 in the Pediatric Neurosurgery Division of Fernandes Figueira Institute-Oswaldo Cruz Foundation (IFF-Fiocruz) in Rio de Janeiro, Brazil. Data were collected from medical records of the neurosurgery outpatient clinic. Only patients with a minimum follow-up of 1 year were included. Patients with congenital infections, other congenital malformations, or intraventricular hemorrhage were excluded. Any patient whose medical records were missing was also excluded. Thus, 231 patients with MMC were included in the analysis. Patients were followed for periods ranging from 1 to 20 years, with a mean of 6.9 years.

Data collection included demographic characteristics (gestational age, sex, and birth weight); clinical features (head circumference at birth, anatomical and functional levels of MMC, hydrocephalus, symptomatic CM-II, neurogenic bladder, and urinary tract infection [UTI]); and surgical details such as timing of repair of MMC, age at first shunt placement, shunt surgery modality (elective or emergency), concurrent surgery (correction of MMC and shunt insertion in the same surgery), incidence and cause of shunt dysfunction, use of external ventricular drain, transfontanelle puncture, surgical wound complications prior to shunting, and endoscopic treatment of hydrocephalus.

Hydrocephalus was defined as a rapidly increasing head circumference and progressive ventriculomegaly on serial ultrasound studies. Macrocephaly was considered when the head circumference was $\geq 38 \mathrm{~cm}$ at birth.

Shunt independence was considered when patients with discontinued ventriculoperitoneal shunts did not present symptoms. We conceive discontinued shunts as occurring when fracture, disconnection, or proximal or distal catheter migration was present.

Symptomatic CM-II in neonates and infants was considered when low cranial neuropathies, swallowing dysfunctions (neurogenic dysphagia), and respiratory disorders (apnea, cyanosis, and laryngeal stridor) were present. In older children, cervicalgia, weakness in the upper limbs, and spasticity were also considered. Clinical dysfunction of swallowing or breathing was confirmed through endoscopy and bronchoscopy.

The anatomical levels were classified according to the radiological records as follows: thoracic (above T12), thoracolumbar (from T12 downward), upper lumbar (L1-2), lower lumbar (L3-5), and sacral. Functionally, because the patients were examined by different professionals, the use of a wide scale to classify all the patients was needed. Therefore, the patients were categorized according to the scale of Hoffer et al., as described in Bartonek et al. ${ }^{2}$ Tethered cord syndrome was defined as back or leg pain, progressive scoliosis, change in bladder function, and motor decline.

Regarding urinary disorders, the patients were categorized into continent without catheterization, socially continent (without losses between catheterization), incontinent, and too young for sphincter control (less than 2 years of age).

We identified the rate of latex allergy. Routinely our population undergoing intermittent clean catheterization for neurogenic bladder uses latex-free catheters. However, the protocol for prevention of latex allergy is only performed in the operating room when there is a confirmed allergy. We evaluated the main causes of hospitalization and death to assess morbidity and mortality.

This project was approved by the Human Research Ethics Committee of IFF-Fiocruz.

\section{Statistical Analysis}

Descriptive analysis was performed to obtain the frequencies and measures of central tendency (mean and median) of the analyzed variables. The bivariate analysis was used to obtain measures of association between the risk factors and main causes of hospitalization and death. For numerical variables the Student t-test was performed when they had a normal distribution, or the Mann-Whitney U-test when they did not. Categorical variables were assessed using the chi-square or Fisher test. The logistic regression model was performed to examine the relationship between the risk factors and main causes of hospitalization and death. Statistically significant differences were determined by $\mathrm{p}$ values $<0.05$. All statistical analyses were performed using standard statistical software (IBM SPSS v22.0, IBM Corp.).

\section{Results}

The main characteristics of our patients are presented in Table 1. Most of the patients of this cohort were born by cesarean section, with a mean gestational age of 37.9 weeks. Only 36 patients (15.6\%) were preterm. There was a discrete predominance of females (55.4\%). Surgical repair of MMC was performed within 48 hours of birth in 157 cases (67.9\%), as shown in Table 1.

\section{Anatomical and Functional Level}

The frequencies of the anatomical levels in decreasing order were as follows: lower lumbar (119), thoracic-lumbar transition (50), upper lumbar (29), sacral (26), and thoracic (7). However, most of the patients presented functionally with thoracic level on the Hoffer scale, representing 37.2\% 
TABLE 1. Characteristics of the cohort of 231 patients with MMC

\begin{tabular}{lc}
\hline \multicolumn{1}{c}{ Characteristic } & Value \\
\hline Gestational age in wks, mean (SD) & $37.9(1.6)$ \\
\hline Birth weight in g, mean (SD) & $3072(580)$ \\
\hline Sex, \% female & 55.4 \\
\hline Age at shunt placement, \% & 38 \\
\hline$<40$ wks & 62 \\
\hline$\geq 40$ wks & $36.5(3.8)$ \\
\hline Head circumference at birth in cm, mean (SD) & 83.5 \\
\hline Hydrocephalus, $\%$ & 47.2 \\
\hline Shunt revisions, \% & 1.73 \\
\hline PFD, \% & 4.8 \\
\hline Tethered cord release, \% & 2.2 \\
\hline ETV, \% & \\
\hline Timing of repair of MMC, \% & 48.9 \\
\hline$<24$ hrs & 19 \\
\hline$<48$ hrs & 12.2 \\
\hline$<72$ hrs & 19.9 \\
\hline$\geq 72$ hrs
\end{tabular}

of the cases (86/231). This functional level was followed by lower lumbar in $28 \%$ (65), upper lumbar in $25.5 \%$ (59), and sacral in $9.1 \%$ (21). Thus, we noticed a tendency for the functional level to be worse than the anatomical level.

\section{Chiari Malformation Type II}

The frequency of symptomatic CM-II was $12 \%$, and it was found mostly in newborns and infants who presented with swallow and respiratory dysfunction. Only 1 patient was older, presenting at the age of 4 years with weakness of upper limbs.

The initial surgical treatment consisted of shunt insertion or revision. In this group, shunt insertion was performed in 26 patients and shunt revisions in 2 cases. Patients whose symptoms did not improve with these measures were candidates for posterior fossa decompression (PFD), which was required in only 4 patients. In 3 of them, PFD was performed within 2 months of age and in 1 patient at 4 years. Two deaths occurred among the 4 patients, one at 12 months due to renal failure and the other at 13 months of age due to shunt dysfunction. After 4 years of age, no patient presented with symptomatic CM-II.

\section{Hydrocephalus}

Two hundred twelve patients (91.8\%) had ventriculomegaly diagnosed at birth by transfontanelle ultrasound. Of these, 193 (83.5\%) were diagnosed with hydrocephalus. Shunt placement was mostly performed after 40 weeks of gestational age (Table 1). Concurrent surgery was performed in 9 patients. All shunt procedures were performed electively. All patients received a standard differential pressure valve.

As shown in Table 2, the frequency of shunt placement was observed mainly among patients with the highest-level MMC ( $\mathrm{p}<0.01)$. Ninety-one of the 193 patients $(47.2 \%)$
TABLE 2. MMC anatomical level and frequency of shunt placement

\begin{tabular}{lccr}
\hline \multirow{2}{*}{ Anatomical Level } & \multicolumn{2}{c}{ Shunt } & \\
\cline { 2 - 3 } & \multicolumn{1}{c}{ Yes } & No & Total \\
\hline Thoracic & $7(100 \%)^{*}$ & $0(0 \%)$ & 7 \\
\hline Thoracolumbar & $47(94 \%)^{*}$ & $3(6 \%)$ & 50 \\
\hline Upper lumbar & $25(86.2 \%)^{*}$ & $4(13.8 \%)$ & 29 \\
\hline Lower lumbar & $100(84 \%)^{*}$ & $19(16 \%)$ & 119 \\
\hline Sacral & $14(53.8 \%)^{*}$ & $12(46.2 \%)$ & 26 \\
\hline Total & $193(83.5)$ & $38(16.5 \%)$ & 231 \\
\hline${ }^{*} p<0.01$. & &
\end{tabular}

with hydrocephalus underwent at least one shunt revision. The first revision occurred on average at 13 months old and the main cause of this procedure was attributed to proximal obstruction (30\%). Multiple shunt revisions were recorded in 34 patients (17.6\%). Shunt infection occurred in $10 \%$ of the cases.

Looking for all demographic, clinical, and surgical variables that could have an influence in shunt dysfunction, we noticed that head circumference $\geq 38 \mathrm{~cm}$ at birth was a significant risk factor for shunt revision $(\mathrm{p}<0.001$; 95\% CI 1.092-1.354). Also, the lumbar functional level of MMC was associated with less revision than upper levels ( $\mathrm{p}<0.014 ; 95 \%$ CI $0.143-0.805)$. These results are shown in Tables 3 and 4.

Endoscopic third ventriculostomy (ETV) was the treatment of choice only in children who presented with hydrocephalus after 1 year of age or in patients with shunt dysfunction and ventricles large enough to allow neuroendoscopy. Thus, the ETV frequency of this series is low; the procedure was performed in only 5 patients. Two of them were previously shunt free and older than 1 year, with a good response to the treatment and with 3 years of follow-up in one case and 2 years in the other. In the remaining cases the patients presented with shunt dysfunction, but ETV was not enough to resolve the clinical condition, and a subsequent shunt revision was needed in all 3 cases.

During follow-up, 10 of 193 patients (5.2\%) undergoing shunt treatment were considered shunt independent. Among them, 6 experienced symptoms of dysfunction of this system and underwent emergency surgery. The mean age at presentation of the symptoms was 13 years and the mean time between the diagnosis of shunt independence and symptoms of dysfunction was 4.7 years.

\section{Tethered Cord Syndrome}

Tethered cord syndrome was diagnosed in 11 patients $(4.8 \%)$ and all of them underwent surgery for spinal cord release. Among them, $45.5 \%$ were wheelchair dependent.

\section{Bladder and Bowel Function and UTls}

Only 2 patients $(0.9 \%)$ were continent without catheterization and 55 (23.8\%) achieved social continence. In contrast, 141 patients $(61 \%)$ presented with urinary incontinence even with intermittent catheterization. Patients 
TABLE 3. Variables used in bivariate analysis for shunt dysfunction

\begin{tabular}{|c|c|c|c|c|c|c|}
\hline \multirow[b]{3}{*}{ Variable } & \multicolumn{4}{|c|}{ Shunt Dysfunction } & \multirow[b]{3}{*}{$p$ Value } & \multirow[b]{3}{*}{ OR $(95 \% \mathrm{Cl})$} \\
\hline & \multicolumn{2}{|c|}{ Yes } & \multicolumn{2}{|c|}{ No } & & \\
\hline & No. & $\%$ & No. & $\%$ & & \\
\hline Timing of repair of MMC & & & & & \multicolumn{2}{|l|}{0.6807} \\
\hline$<24 \mathrm{hrs}$ & 50 & $51.0 \%$ & 48 & $49.0 \%$ & & Reference \\
\hline$<48 \mathrm{hrs}$ & 18 & $50.0 \%$ & 18 & $50.0 \%$ & & $1.042(0.485-2.236)$ \\
\hline$<72 \mathrm{hrs}$ & 8 & $40.0 \%$ & 12 & $60.0 \%$ & & $1.563(0.587-4.156)$ \\
\hline$\geq 72 \mathrm{hrs}$ & 15 & $41.7 \%$ & 21 & $58.3 \%$ & & $1.458(0.674-3.156)$ \\
\hline Concurrent surgery & & & & & 0.2558 & \\
\hline Yes & 6 & $66.7 \%$ & 3 & $33.3 \%$ & & $2.329(0.565-9.597)$ \\
\hline No & 85 & $46.2 \%$ & 99 & $53.8 \%$ & & Reference \\
\hline Transfontanelle puncture & & & & & 0.6288 & \\
\hline Yes & 8 & $53.3 \%$ & 7 & $46.7 \%$ & & $1.308(0.455-3.761)$ \\
\hline No & 83 & $46.6 \%$ & 95 & $53.4 \%$ & & Reference \\
\hline Wound complications & & & & & 0.7663 & \\
\hline Yes & 12 & $50.0 \%$ & 12 & $50.0 \%$ & & $1.141(0.485-2.685)$ \\
\hline No & 78 & $46.7 \%$ & 89 & $53.3 \%$ & & Reference \\
\hline Anatomical level of MMC & & & & & 0.285 & \\
\hline Thoracic & 4 & $57.1 \%$ & 3 & $42.9 \%$ & & $3.000(0.446-20.15)$ \\
\hline Upper lumbar & 39 & $54.2 \%$ & 33 & $45.8 \%$ & & $2.659(0.750-9.428)$ \\
\hline Lower lumbar & 43 & $43.0 \%$ & 57 & $57.0 \%$ & & $1.697(0.490-5.880)$ \\
\hline Sacral & 4 & $30.8 \%$ & 9 & $69.2 \%$ & & Reference \\
\hline Functional level of MMC & & & & & 0.0026 & \\
\hline Thoracic & 50 & $61.0 \%$ & 32 & $39.0 \%$ & & $6.25(1.247-31.320)$ \\
\hline Lumbar & 36 & $38.7 \%$ & 57 & $61.3 \%$ & & $2.526(0.507-12.570)$ \\
\hline Sacral & 2 & $20.0 \%$ & 8 & $80.0 \%$ & & Reference \\
\hline Gestational age in wks ${ }^{*}$ & 88 & $38.2(1.4)$ & 98 & $37.9(1.5)$ & 0.229 & $0.886(0.727-1.080)$ \\
\hline Weight in g† & 90 & $1.9(0.3)$ & 102 & $1.8(0.4)$ & 0.511 & $0.765(0.346-1.695)$ \\
\hline Head circumference $\geq 38 \mathrm{~cm} \ddagger$ & 85 & $38.2(4.4)$ & 99 & $35.6(2.9)$ & 0.001 & $0.812(0.736-0.894)$ \\
\hline
\end{tabular}

who were not old enough for their sphincter control to be evaluated corresponded to 33 cases (14.3\%).

Correlating the functional level with the degree of continence, it is evident that urinary incontinence is present in high frequency at all functional levels and, therefore, there is no relation between the neurogenic bladder and

TABLE 4. Logistic regression model prognostic factors for shunt dysfunction and revision

\begin{tabular}{ccc}
\hline Variable & $\mathrm{p}$ Value & OR $(95 \% \mathrm{Cl})$ \\
\hline Functional level of MMC & 0.035 & - \\
\hline Thoracic & 0.267 & $0.64(0.290-1.408)$ \\
\hline Lumbar & $0.014^{*}$ & $0.34(0.143-0.805)$ \\
\hline Sacral & 0.054 & $0.12(0.014-1.038)$ \\
\hline Head circumference $\geq 38 \mathrm{~cm}$ & $0.001^{*}$ & $1.2(1.092-1.354)$ \\
\hline
\end{tabular}

* Statistically significant at $p<0.05$. the MMC level (Fig. 1). Likewise, homogeneous distribution was found in cases of chronic UTIs; it was present in 129 patients (55.8\%). However, there was a significant association between recurrent UTI and thoracic functional level (Table 5).

Bowel dysfunction was present in the majority of the cohort, with 169 patients (73.2\%) presenting with fecal incontinence and 111 (48.1\%) presenting with constipation.

\section{Latex Allergies}

Latex allergy occurred in 7 patients (3\%). Of these, 6 were female. All patients underwent intermittent catheterization due to neurogenic bladder. Only 1 patient had a history of excessive exposure to latex, with 3 shunt revisions, 1 vesicostomy, and 5 hospitalizations due to UTI.

\section{Hospitalization and Mortality}

In this series there were 314 hospitalizations (mean: 


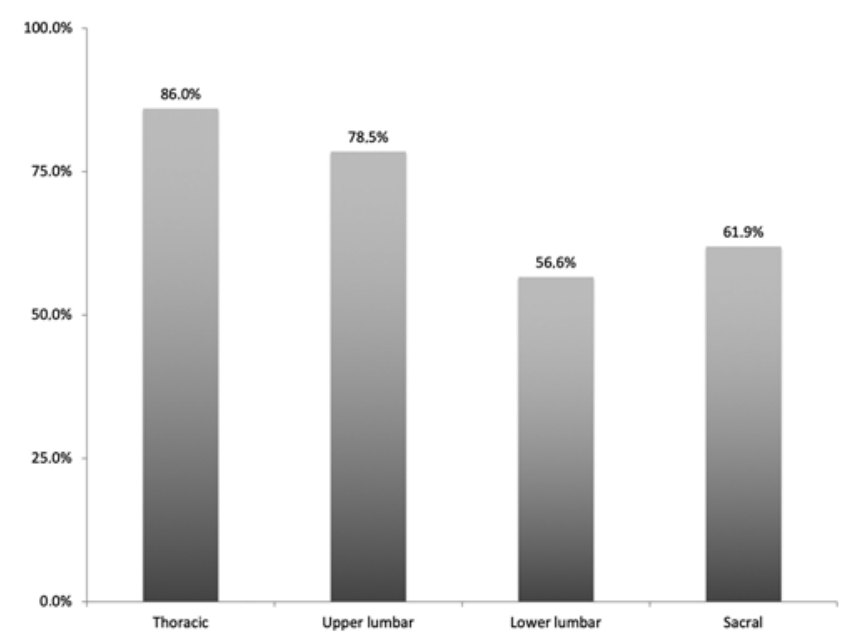

FIG. 1. Bar graph showing distribution (\%) of neurogenic bladder between functional levels of MMC.

2 hospitalizations per patient). Most of them were attributed to shunt dysfunction, in 118 cases (37.6\%), followed by UTI in 56 (18\%), and suspected but unconfirmed shunt dysfunction in $16(5 \%)$. Other causes of hospitalization were (in order of frequency): pressure ulcers, tethered cord syndrome, epilepsy, pneumonia, and others.

Seventeen deaths (7.4\%) were noted. Of these, $6(35 \%)$ were attributed to shunt dysfunction, 5 of which were due to infection and 1 due to proximal obstruction. The second cause of death was urinary sepsis and renal failure, corresponding to 5 children. The other deaths were identified through the information registry of the Rio de Janeiro Court, with unknown cause.

Considering causes of hospitalization and deaths as prognostic indicators, shunt dysfunction and UTIs were the 2 main features responsible for morbidity and mortality.

\section{Discussion}

The survival rate of patients with MMC has increased due to advances in the treatment of the associated conditions, ${ }^{3,4,25}$ such as hydrocephalus and neurogenic bladder. Thus, multidisciplinary and specialized attention is required throughout life. ${ }^{19}$

The Pediatric Neurosurgery Division of the IFF-Fiocruz is a reference center that coordinates numerous specialists involved in the long-term treatment of patients with MMC. In our series, ventriculomegaly was evident in 212 patients and treatment for hydrocephalus was required in 193 (83.5\%) of the cases, as shown by previously reported data in the literature. ${ }^{4,10}$

We evaluated the main causes of hospitalization and death to assess morbidity and mortality. In this way we found that shunt dysfunction and UTI were the 2 main features responsible for morbidity and mortality. Previously, the prognosis of these children was assessed based on patient survival. ${ }^{32}$ Nowadays, shunt device survival is considered the basis for determining the prognosis, because the rate of shunt dysfunction is relatively high in patients with
TABLE 5. Functional level of MMC and recurrent UTI

\begin{tabular}{lcrr}
\hline & \multicolumn{2}{c}{ UTI } & \\
\cline { 2 - 3 } Functional Level & \multicolumn{1}{c}{ Yes } & No & Total \\
\hline Thoracic & $54(62.8 \%)^{*}$ & $32(37.2 \%)$ & 86 \\
\hline Upper lumbar & $29(49.2 \%)$ & $30(50.8 \%)$ & 59 \\
\hline Lower lumbar & $34(52.3 \%)$ & $31(47.7 \%)$ & 65 \\
\hline Sacral & $12(57.1 \%)$ & $9(42.9 \%)$ & 21 \\
\hline Total & $129(55.8 \%)$ & $102(44.2 \%)$ & 231 \\
\hline${ }^{*} p<0.025$. & &
\end{tabular}

MMC ${ }^{6,9,30}$ Bowman et al. ${ }^{4}$ have shown that the mortality rate continues to rise as patients approach adulthood, with the main cause being shunt dysfunction. As previously described, ${ }^{4}$ we observed a high rate of shunt dysfunction (47.2\%). This occurred on average at 13 months of life.

There is evidence in the literature that some features could lead to shunt dysfunction, such as gestational age, birth weight, and age at shunt placement. ${ }^{35}$ Looking forward using the logistic regression model (Table 4), in our series these features were not related to the risk of shunt dysfunction.

Regarding the timing of repair of the spinal defect, because cesarean sections were usually scheduled, repair of MMC occurred in the first 48 hours of life in $67.9 \%$ of the cases, the majority of them being performed in the first 24 hours. Late repairs occurred only in cases of nonscheduled deliveries, when prenatal complications were present. In the literature, there is a recommendation for the treatment of MMC within the first 48 or 72 hours. ${ }^{5,21}$ An increased risk of shunt revision was reported after late MMC repair, due to the increase in circulating proteins and debris in CSF, which would cause shunt obstruction, even without infection. ${ }^{12,34}$ In this cohort, we found no association between late repair of MMC and the frequency of shunt revisions (Table 3). The absence of this association can be explained by the adequate care of the newborn (wound protection and antibiotic therapy). Despite this, we recommend that the MMC repair be done as early as possible.

There is controversy in the literature regarding the risk of complications after concurrent repair of MMC and shunt insertion. Some authors ${ }^{18}$ did not demonstrate a significant increase in complications, although others ${ }^{26}$ demonstrated risks related to CSF exposure, transient bacteremia during the surgical manipulation of MMC, and unrecognized UTIs. In our series, concurrent surgeries were performed only when considerable macrocephaly was present at birth and we did not demonstrate an increased risk of shunt failure in relation to concurrent surgery.

Oakeshott and Hunt ${ }^{24}$ demonstrated that children with a head circumference above the 90th percentile had a worse prognosis. In a recent cohort a dismal prognosis related to a large head circumference was also described. ${ }^{17}$ Our results corroborate these studies and, as far as we know, for the first time in the literature we demonstrate the association of a cutoff point of $38 \mathrm{~cm}$ of head circumference at birth with increased risk of shunt dysfunction (Table 4). The Management of Myelomeningocele Study (MOMS) $)^{36}$ also showed a worse prognosis in patients with exuberant 
ventricular dilatation ( $\geq 15 \mathrm{~mm}$ ), and it is not possible to avoid shunting in these cases. For these patients, possibly the best treatment would continue to be postnatal surgery, preserving greater maternal and fetal safety. Also, as reported before, ${ }^{17}$ we noticed that the incidence of hydrocephalus varies according to the anatomical level of the spinal defect and that shunt dysfunction is more common among the highest levels of the defect (Tables 2 and 3). This fact should be considered when comparing the prognosis of fetal intervention and postnatal repair of MMC.

The shunt independence concept is based on the context of arrested hydrocephalus. Hurni et al..$^{14}$ describe circumstances in which hydrocephalus can be spontaneously arrested. In such a situation, ventricular enlargement is no longer active, intracranial pressure returns to normal values, and a conservative approach is often adopted. In this context, Iannelli et al. ${ }^{15}$ reported in a retrospective series of 850 children with nontumoral hydrocephalus that shunt independence could be demonstrated in $3.2 \%$ of the patients (27 cases). In our series, we initially considered 10 patients $(5.2 \%)$ to be shunt independent, but in more than half of them $(60 \%)$ symptoms of dysfunction worsened and the patients underwent prompt surgery. Talamonti et al. ${ }^{34}$ also initially considered 34 patients (24\%) with MMC to be shunt independent. However, 10 patients throughout the follow-up required shunt revision. Other authors have reported that a disconnected portion of fractured shunt may be working and that it is not safe to state that the shunt is no longer needed. ${ }^{11,28}$ In this way, we believe that the concept of arrested hydrocephalus should be treated with great care and that patients with catheter fractures, disconnections, or migrations should be carefully followed up with routine control examinations, and also that the ability of the family to access the hospital should be considered in the decision between surgical or conservative treatment.

UTI was the second most common cause of morbidity and mortality. As expected, ${ }^{29}$ virtually all patients presented with neurogenic bladder, with a homogeneous distribution of incontinent patients between the thoracic, lumbar, and sacral levels. The same distribution was demonstrated for recurrent UTIs, with the exception of the thoracic functional level, which correlated with a higher number of UTIs (Table 5). The urodynamic protocol for patients with recurrent UTIs consists of prophylactic antibiotic therapy, increased frequency of intermittent catheterizations, and early vesicostomy when needed. This protocol usually allows the control of infections, which makes us think that possibly nonadherence to treatment may be a limiting factor for the prevention of UTIs, but our study did not propose this investigation.

Because the inclusion criteria in this study consisted of a minimum follow-up of 1 year, we evaluated the later symptoms of CM-II. As observed in other series, ${ }^{4,34}$ the frequency of CM-II symptoms was approximately $12 \%$. The initial surgical treatment usually consists of shunt insertion or revision. ${ }^{33}$ Patients whose symptoms do not improve with these measures are candidates for PFD. Our results are compatible with the literature. We report shunt insertion in 26 cases and shunt revisions in 2 cases. PFD was required in only 4 patients; in 3 of them PFD occurred within 2 months of birth, and in 1 it occurred at 4 years of age. Among these 4 patients, 2 deaths occurred, one at 12 months due to renal failure and the other at the age of 13 months due to shunt dysfunction-these were our most important indicators of morbidity and mortality. We also emphasize that after 4 years no patient presented with the symptomatic form of CM-II.

As previously reported, ${ }^{27}$ we noticed that the functional level tended to be worse than the anatomical level. However, because this is a retrospective study, many different professionals performed the clinical examination. Thus, a wide scale was used to classify all patients, and therefore there may be some bias. In addition, in the literature there are reports of poor clinical outcome leading to motor worsening, ${ }^{7}$ which may explain the discordance found.

According to the available literature, ${ }^{4}$ only $10 \%-30 \%$ of patients develop tethered cord syndrome. Our results are compatible with the reported data, with a frequency of $4.8 \%$. The mean age of the patients was 7.1 years, although 2 peaks of incidence were reported in the literature.

Several authors ${ }^{8,22,23}$ have reported that latex allergy depends on the degree of exposure to it, due to multiple surgical procedures. In the Bowman et al. series, ${ }^{4}$ approximately one-third of the cohort presented with a latex allergy. Interestingly, our results contradict the findings in the literature. Usually we did not use a latex-free protocol in the operating room, and yet only 7 patients (3\%) were identified as allergic. Of these, only 1 had a history of excessive exposure to the allergen. Our results led to the understanding that repeated exposure alone is not enough to trigger the allergic reaction and that it may also depend on individual predisposition.

This long-term cohort allows us to know better the population of survivors and to prevent possible events that increase the rate of hospitalization and morbidity and mortality, aiming to provide a better quality of life. Even though the 2 main causes of morbidity and mortality have been described in the literature, ${ }^{4,24,25}$ the mortality of the group of patients that we studied is lower, demonstrating that we are moving in the right direction.

\section{Conclusions}

Myelomeningocele is the most complex malformation of the CNS compatible with long-term survival. Morbidity and mortality, previously associated with CM-II, now are related to shunt dysfunction and frequency of UTIs. Macrocephaly at birth and higher levels of the defect have an impact on worse outcome, related to more shunt dysfunctions and UTIs, and therefore are a challenge to the daily practice of pediatric neurosurgery. Also, the concept of shunt independence could be related to sudden shunt dysfunction and should be carefully analyzed. Knowledge of these factors allows us to predict the prognosis, prevent complications, and guide the family and the patient about care and attention throughout life.

\section{References}

1. Albright AL, Pollack IF, Adelson PD: Principles and Practice of Pediatric Neurosurgery, ed 2. New York: Thieme, 2008

2. Bartonek A, Saraste H, Knutson LM: Comparison of dif- 
ferent systems to classify the neurological level of lesion in patients with myelomeningocele. Dev Med Child Neurol 41:796-805, 1999

3. Bowman RM, Boshnjaku V, McLone DG: The changing incidence of myelomeningocele and its impact on pediatric neurosurgery: a review from the Children's Memorial Hospital. Childs Nerv Syst 25:801-806, 2009

4. Bowman RM, McLone DG, Grant JA, Tomita T, Ito JA: Spina bifida outcome: a 25-year prospective. Pediatr Neurosurg 34:114-120, 2001

5. Bulbul A, Can E, Bulbul LG, Cömert S, Nuhoglu A: Clinical characteristics of neonatal meningomyelocele cases and effect of operation time on mortality and morbidity. Pediatr Neurosurg 46:199-204, 2010

6. Caldarelli M, Di Rocco C, La Marca F: Shunt complications in the first postoperative year in children with meningomyelocele. Childs Nerv Syst 12:748-754, 1996

7. Coniglio SJ, Anderson SM, Ferguson JE II: Functional motor outcome in children with myelomeningocele: correlation with anatomic level on prenatal ultrasound. Dev Med Child Neurol 38:675-680, 1996

8. De Swert LF, Van Laer KM, Verpoorten CM, Van Hoeyveld EM, Cadot P, Stevens EA: Determination of independent risk factors and comparative analysis of diagnostic methods for immediate type latex allergy in spina bifida patients. Clin Exp Allergy 27:1067-1076, 1997

9. Di Rocco C, Marchese E, Velardi F: A survey of the first complication of newly implanted CSF shunt devices for the treatment of nontumoral hydrocephalus. Cooperative survey of the 1991-1992 Education Committee of the ISPN. Childs Nerv Syst 10:321-327, 1994

10. Dias MS, McLone DG: Hydrocephalus in the child with dysraphism. Neurosurg Clin N Am 4:715-726, 1993

11. Erol FS, Ozturk S, Akgun B, Kaplan M: Ventriculoperitoneal shunt malfunction caused by fractures and disconnections over 10 years of follow-up. Childs Nerv Syst 33:475-481, 2017

12. Gamache FW Jr: Treatment of hydrocephalus in patients with meningomyelocele or encephalocele: a recent series. Childs Nerv Syst 11:487-488, 1995

13. Hunt GM, Oakeshott P: Outcome in people with open spina bifida at age 35: prospective community based cohort study. BMJ 326:1365-1366, 2003

14. Hurni Y, Poretti A, Schneider J, Guzman R, Ramelli GP: Arrested hydrocephalus in childhood: case series and review of the literature. Neuropediatrics 49:302-309, 2018

15. Iannelli A, Rea G, Di Rocco C: CSF shunt removal in children with hydrocephalus. Acta Neurochir (Wien) 147:503507, 2005

16. Kaufman BA, Terbrock A, Winters N, Ito J, Klosterman A, Park TS: Disbanding a multidisciplinary clinic: effects on the health care of myelomeningocele patients. Pediatr Neurosurg 21:36-44, 1994

17. Kellogg R, Lee P, Deibert CP, Tempel Z, Zwagerman NT, Bonfield CM, et al: Twenty years' experience with myelomeningocele management at a single institution: lessons learned. J Neurosurg Pediatr 22:439-443, 2018

18. Machado HR, Santos de Oliveira R: Simultaneous repair of myelomeningocele and shunt insertion. Childs Nerv Syst 20:107-109, 2004

19. Mattsson S, Gladh G: [Children with meningomyelocele become adults!] Lakartidningen 102:2566-2570, 2005 (Swedish)

20. McLone DG: Treatment of myelomeningocele: arguments against selection. Clin Neurosurg 33:359-370, 1986

21. Messing-Jünger M, Röhrig A: Primary and secondary management of the Chiari II malformation in children with myelomeningocele. Childs Nerv Syst 29:1553-1562, 2013

22. Michael T, Niggemann B, Moers A, Seidel U, Wahn U,
Scheffner D: Risk factors for latex allergy in patients with spina bifida. Clin Exp Allergy 26:934-939, 1996

23. Niggemann B, Buck D, Michael T, Wahn U: Latex provocation tests in patients with spina bifida: who is at risk of becoming symptomatic? J Allergy Clin Immunol 102:665670, 1998

24. Oakeshott P, Hunt GM: Long-term outcome in open spina bifida. Br J Gen Pract 53:632-636, 2003

25. Oakeshott P, Hunt GM, Poulton A, Reid F: Open spina bifida: birth findings predict long-term outcome. Arch Dis Child 97:474-476, 2012

26. Pang D: Surgical complications of open spinal dysraphism. Neurosurg Clin N Am 6:243-257, 1995

27. Rintoul NE, Sutton LN, Hubbard AM, Cohen B, Melchionni J, Pasquariello PS, et al: A new look at myelomeningoceles: functional level, vertebral level, shunting, and the implications for fetal intervention. Pediatrics 109:409-413, 2002

28. Roth J, Constantini S: The disconnected shunt: a window of opportunities. Childs Nerv Syst 33:467-473, 2017

29. Sager C, Burek C, Corbetta JP, Weller S, Ruiz J, Perea R, et al: Initial urological evaluation and management of children with neurogenic bladder due to myelomeningocele. J Pediatr Urol 13:271.e1-271.e5, 2017

30. Sainte-Rose C: Shunt obstruction: a preventable complication? Pediatr Neurosurg 19:156-164, 1993

31. Salomão JF, Bellas AR, Leibinger RD, Barbosa AP, Brandão MA: [Symptomatic Chiari type II malformation.] Arq Neuropsiquiatr 56:98-106, 1998 (Portuguese)

32. Shurtleff DB, Kronmal R, Foltz EL: Follow-up comparison of hydrocephalus with and without myelomeningocele. J Neurosurg 42:61-68, 1975

33. Stevenson KL: Chiari Type II malformation: past, present, and future. Neurosurg Focus 16(2):E5, 2004

34. Talamonti G, D'Aliberti G, Collice M: Myelomeningocele: long-term neurosurgical treatment and follow-up in 202 patients. J Neurosurg 107 (5 Suppl):368-386, 2007

35. Tuli S, Drake J, Lawless J, Wigg M, Lamberti-Pasculli M: Risk factors for repeated cerebrospinal shunt failures in pediatric patients with hydrocephalus. J Neurosurg 92:31-38, 2000

36. Tulipan N, Wellons JC III, Thom EA, Gupta N, Sutton LN, Burrows PK, et al: Prenatal surgery for myelomeningocele and the need for cerebrospinal fluid shunt placement. J Neurosurg Pediatr 16:613-620, 2015

\section{Disclosures}

The authors report no conflict of interest concerning the materials or methods used in this study or the findings specified in this paper.

\section{Author Contributions}

Conception and design: T Protzenko, JFM Salomão, Gomes. Acquisition of data: T Protzenko, Fontes. Analysis and interpretation of data: T Protzenko, Bellas, Fontes, JFM Salomão, Gomes. Drafting the article: T Protzenko, JFM Salomão, Gomes. Critically revising the article: Bellas, Pousa, M Protzenko, Fontes, Silveira, Sá, Pereira, JFM Salomão, Gomes. Reviewed submitted version of manuscript: Pousa, M Protzenko, Fontes, Silveira, Sá, Pereira, JFM Salomão, Gomes. Statistical analysis: Gomes. Administrative/technical/material support: Bellas, RM Salomão. Study supervision: Bellas, JFM Salomão, Gomes.

\section{Correspondence}

Tatiana Protzenko: Fernandes Figueira National Institutes of Health for Women, Children, and Adolescents, Oswaldo Cruz Foundation (IFF-Fiocruz), Rio de Janeiro, Brazil. tatiana. protzenko@gmail.com. 